\title{
Análise da produção científica brasileira sobre badminton: uma revisão integrativa
}

\section{Analysis of Brazilian scientific production about badminton: an integrative review}

\author{
Patric Paludett Flores ${ }^{1}$, Ana Luíza Barbosa Anversa ${ }^{2}$, Leonardo Cordeiro de Queiroz ${ }^{2}$, \\ Fernando Lazaretti Onorato Silva ${ }^{2}$, Ariel Arthur Cardoso dos Santos Coutinho², \\ Vânia de Fátima Matias de Souza² \\ ${ }^{1}$ Universidade Estadual do Paraná (Unespar), Paranavaí/PR, Brasil \\ 2 Universidade Estadual de Maringá (UEM), Maringá/PR, Brasil
}

\author{
HISTÓRICO DO ARTIGO \\ Recebido: 18 abril 2020 \\ Revisado: 29 julho 2020 \\ Aprovado: 31 julho 2020
}

\section{PALAVRAS-CHAVE:}

Esporte de Raquete; Badminton; Produção Científica.

\section{KEYWORDS:}

Racket Sport; Badminton; Scientific Production.

\section{RESUMO}

INTRODUÇÃO: A presente pesquisa de revisão integrativa, teve como foco o esporte Badminton, modalidade que integra a família dos esportes de raquete e que tem se difundido nas últimas décadas devido ao seu caráter motivador e participativo social.

OBJETIVO: Analisar a produção científica nacional online sobre o esporte Badminton a partir da busca nas bases de dados Lilacs, Medline, Portal Scielo e Portal de Periódicos CAPES.

MÉTODOS: Para o levantamento dos artigos nas bases de dados mencionadas, utilizou-se em um primeiro levantamento o descritor: Badminton; e em um segundo momento os descritores: Badminton "and" Esportes de Raquete. Identificou-se um número de 17325 produções científicas, as quais foram analisadas a partir de critérios de inclusão e exclusão, resultando ao final a seleção de 13 artigos. A interpretação dos dados se constituiu pela técnica de análise de conteúdo.

RESULTADOS: Após a leitura na íntegra dos artigos, emergiram três categorias de apresentação dos resultado: a) Mapeamento dos autores da produção selecionada sobre Badminton; sendo possível verificar que os autores possuem vínculo com a temática, a formação inicial dos mesmos se dá no contexto da educação física, fisioterapia e medicina e com destaque para as regiões Nordeste e Sudeste, sendo a Universidade Estadual de Campinas a instituição com o maior número de pesquisadores sobre a temática; b) Procedimentos metodológicos abordados na produção selecionada sobre Badminton; sendo encontrada o predomínio de pesquisa do tipo quantitativo e a coleta dos dados a partir de testes físicos e; c) Enfoque temático das pesquisas selecionadas sobre Badminton, emergindo as temáticas esporte de rendimento e esporte educacional.

CONCLUSÃO: A produção científica sobre Badminton apresenta maior número de autores e artigos nas regiões Nordeste e Sudeste, com pesquisas norteadas sob o método quantitativo e direcionadas, em sua maioria, para o contexto do esporte de rendimento. Deste modo, nota-se que a temática carece de estudos voltados para o esporte educacional, uma vez que foi pouco explorado, bem como para a prática do Badminton na área da saúde e do lazer (esporte de participação), categoria que não aparece na produção científica analisada.

\section{ABSTRACT}

BACKGROUND: The present research of integrative review, focused on the sport Badminton, modality that integrates the family of racket sports and that has spread in the last decades due to its motivating and social participative character.

OBJECTIVE: Analyze the national scientific production online about the sport Badminton from the search in the databases Lilacs, Medline, Portal Scielo and Portal de Periódicos CAPES.

METHODS: For the survey of articles in the mentioned databases, the descriptor was used in a first survey: Badminton; and in a second moment the descriptors: Badminton "and" Racket Sports. A number of 17325 scientific productions were identified, which were analyzed based on inclusion and exclusion criteria, resulting in the selection of 13 articles at the end. The interpretation of the data was constituted by the technique of content analysis.

RESULTS: After reading the articles in full, three categories of presentation of the results emerged: a) Mapping the authors of the selected production on Badminton; it being possible to verify that the authors have a link with the theme, their initial training takes place in the context of physical education, physiotherapy and medicine and with emphasis on the Northeast and Southeast regions, with the State University of Campinas being the institution with the largest number researchers on the theme; b) Methodological procedures covered in the selected production on Badminton; the predominance of quantitative research and data collection based on physical tests was found; c) Thematic focus of selected research on Badminton, with the emergence of performance sports and educational sports.

CONCLUSION: The scientific production on Badminton presents a greater number of authors and articles in the Northeast and Southeast regions, with research guided under the quantitative method and directed, in the majority, to the context of performance sport. Thus, it is noted that the theme lacks studies focused on educational sport, since it was little explored, as well as for the practice of Badminton in the area of health and leisure (sport of participation), a category that does not appear in the scientific production analyzed. 


\section{INTRODUÇÃO}

Os esportes com rede divisória ou parede são modalidades em que o objeto de interação da dinâmica do jogo (bola ou peteca) é arremessado, lançado ou batido em direção à quadra adversária de modo com que o oponente não consiga defendê-lo ou devolvê-lo adequadamente (GONZÁLEZ; DARIDO; OLIVEIRA, 2017). Dentre as modalidades com essas características encontramos o Voleibol, Vôlei de Praia, Tênis de Campo, Tênis de Mesa, Peteca, Squash, Badminton, entre outros.

Para este estudo, toma-se como foco o Badminton, modalidade que tem se difundido nas últimas décadas devido ao seu caráter motivador e participativo social, apresentando as mesmas regras para o esporte convencional e adaptado (STRAPASSON; DUARTE; PEREIRA, 2015; GINCIENE; ABURACHID, 2017).

Este secular jogo de peteca, inicialmente conhecido como Poona na Índia, foi regulamentado na Inglaterra e adquiriu uma legião de apreciadores, em especial após a estreia nas Olimpíadas de Barcelona, em 1992 (SESI, 2012). Pelas estimativas, o Badminton é um esporte praticado por milhões de pessoas em todos os continentes, sendo considerado o segundo esporte mais praticado no mundo (GOMES-DA-SILVA; SOUSA-CRUZ; ARRUDA, 2019).

No Brasil esse esporte ainda é pouco conhecido, diferentemente de alguns países europeus e asiáticos, nos quais a modalidade faz parte da cultura assim como o futebol se faz presente na vida dos brasileiros (ANVERSA; FLORES, 2019). Nesta direção, nota-se que o Badminton, mesmo sendo regulamentado como prática competitiva no país desde o ano de 1980, ainda é uma prática esportiva pouco apreciada nas escolas, por meio da educação física escolar, bem como nos cenários pré-desportivos das cinco regiões do território nacional.

Contudo, nos últimos anos, tem-se notado uma procura e um crescimento razoável de praticantes de Badminton no país, além disso, atualmente, o Brasil conta com fortes atletas Olímpicos e Paralímpicos na modalidade (CBBD, 2020). Um dos fatores que pode ter influenciado esta ação é o fato de o Brasil ter vivenciado um momento único no campo esportivo, ao ser sede, em 2016 no Rio de Janeiro, dos Jogos Olímpicos. A oportunidade, presenciou e trouxe visibilidade a inúmeras modalidades esportivas pouco difundidas por aqui, como por exemplo o Badminton, configurando-se como um momento ímpar e possibilitando que tal modalidade se tornasse mais conhecida e explorada enquanto esporte de rendimento, bem como uma prática corporal em potencial no dia a dia da população.

Neste sentido, percebe-se que o Badminton vem ganhando destaque no cenário brasileiro, não apenas como uma modalidade esportiva de rendimento, mas como também uma atividade de caráter educacional e/ou de saúde e lazer, considerado esporte de participação (ANVERSA; FLORES, 2019). Movimento que não é diferente em outras modalidades, visto que, conforme Bento (2014), o esporte moderno de forma geral, enquanto manifestação cultural, vem adquirindo novos sentidos e significados, passando a ser visto de forma polissêmica, ao trazer em sua essência uma diversidade de sentidos e formas, o que o torna um fenômeno mais amplo e plural, transcendendo seu caráter de apenas competição.

Essa conjuntura polissêmica do esporte é uma disposição que também tende a refletir no subcampo científico. Assim, "[...] os diferentes contornos apresentados pelas manifestações esportivas apresentam peculiaridades a serem levantadas, investigadas e analisadas pelo universo científico a fim de que se compreenda o fenômeno na sua complexidade" (SOUZA; SILVA; MOREIRA, 2016, p. 1106). Nesta direção, a presente pesquisa tem por objetivo analisar a produção científica nacional online sobre o esporte Badminton a partir da busca nas bases de dados Lilacs, Medline, Portal Scielo e Portal de Periódico CAPES. Para atingir ao objetivo, tomaram-se como base as seguintes questões norteadoras: Quais os principais autores e centros de produção dos artigos sobre a temática? Quais os procedimentos metodológicos adotados nas pesquisas encontradas? Quais os principais enfoques temáticos dos artigos publicados?

\section{MÉTODOS}

Esta pesquisa se caracteriza como uma revisão integrativa de literatura, uma vez que busca sintetizar resultados de pesquisas anteriores sobre o tema, de modo a sistematizar os achados das pesquisas na compreensão de um fenômeno específico, relacionada à questão investigativa (CROSSETTI, 2012). Deste modo, para a autora, o pesquisador pode construir uma revisão integrativa de literatura com diferentes finalidades, as quais podem traçar a definição de conceitos, revisão de teorias ou análise metodológica de pesquisas sobre um tópico em particular e a partir da comparação dos dados obter as conclusões gerais sobre o problema de pesquisa.

Para definir a produção científica a ser explorada no estudo, inicialmente, foi realizado por três pesquisadores, de modo individual, um levantamento dos artigos científicos nas bases de dados: Lilacs e Medline, por meio da interface da Biblioteca Virtual da Saúde ${ }^{1}$ (BVS - Bireme); Portal Scielo ${ }^{2}$ e Portal de Periódicos ${ }^{3}$ da CAPES. Ressalta-se que para realizar a busca da produção foco deste estudo, os pesquisadores utilizaram como descritor em um primeiro momento: Badminton e; em um segundo momento, a combinação dos termos: Badminton "and" Esporte de Raquete. Após a pré-seleção dos artigos, por parte de cada um dos pesquisadores, foi realizada a discussão e comparação, em grupo, da produção levantada.

Nesta direção, foram encontrados um total de 17.325 artigos, os quais foram analisados seguindo critérios de inclusão e exclusão. Destaca-se que inicialmente a análise se constituiu por meio da leitura do título, resumo e palavras-chave e, após tal ação, foi realizada a leitura na íntegra dos textos. Para o artigo ser incluído, ele precisou ser uma pesquisa que aborda apenas a modalidade Badminton; ser um artigo original; e, ser uma pesquisa realizada no cenário brasileiro. A produção que não atendeu aos critérios acima descritos, foi excluída por apresentar os seguintes motivos: a) pesquisas que abordam as modalidades de esportes de raquete de maneira geral; b) produção de caráter de revisão, ensaios, resenhas, teses, dissertações ou anais de evento; c) artigos que retratam o badminton fora do cenário brasileiro; e, d) artigos repetidos nas bases de dados. Destaca-se que para este estudo não foi considerado um período temporal de análise, visto que a ideia foi abarcar o maior número de produções sobre a temática no cenário brasileiro.

\footnotetext{
${ }^{1}$ Ver em: www.bvsalud.org

${ }^{2}$ Ver em: www.scielo.org/php/index.php

${ }^{3}$ Ver em: https://www.periodicos.capes.gov.br/
} 
Sendo assim, após o levantamento e análise das produções (Figura 1), foram selecionados 13 artigos, os quais se configuram como a produção científica explorada nesta pesquisa.

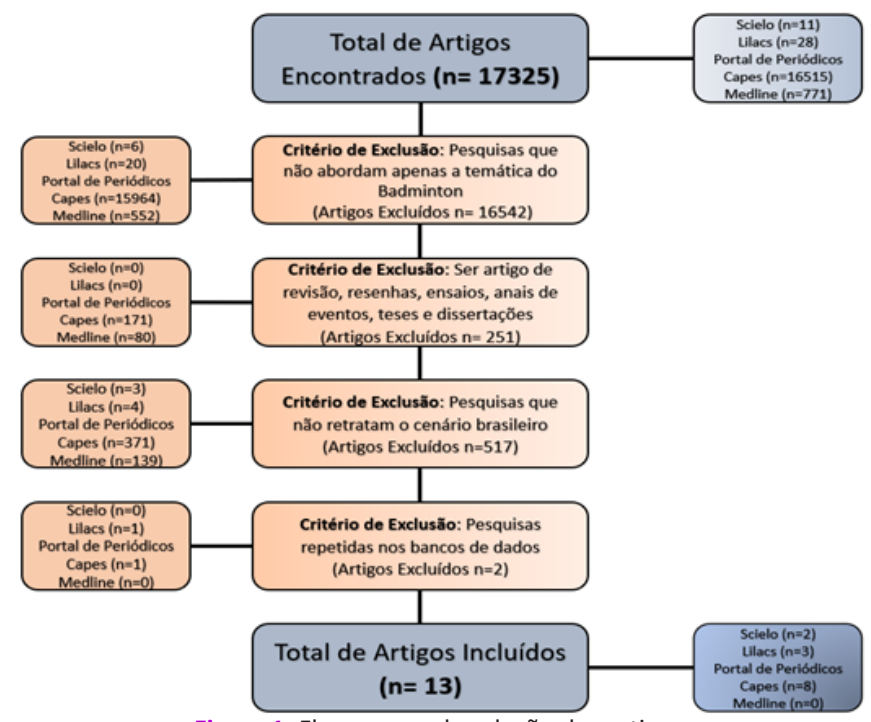

Figura 1. Fluxograma da seleção dos artigos. Fonte: os autores.

Após a seleção da produção, identificou-se os seguintes artigos: Artigo 1 - "Para-Badminton: quantificação dos fundamentos do jogo através do scout" (STRAPASSON et al., 2017); Artigo 2 - "Errar menos para ganhar mais: uma análise no Badminton" (CHIMINAZZO et al., 2017), ambos publicados na Revista Brasileira de Ciência e Movimento; Artigo 3 - "Avaliação das alterações posturais de atletas de Badminton após Stretching Global Ativo" (DANTAS et al., 2014), publicado na Revista ConScientiae Saúde; Artigo 4 - "Badminton: possibilidades de ensino aplicadas ao contexto da Educação Física escolar" (ABURACHID et al., 2019), publicado no Journal of Physical Education; Artigo 5 - "Influência do nível de desempenho de jogadores de Badminton em aspectos neuromotores durante uma tarefa de apontar um alvo" (LOUREIRO JÚNIOR; FREITAS, 2012), publicado na Revista Brasileira de Medicina do Esporte; Artigo 6 - "Entre o lance e a chance: lógica interna numa final de Badminton" (GOMES-DA-SILVA; SOUSA-CRUZ; ARRUDA, 2019), publicado na Revista Motrivivência; Artigo 7 - "Hábito alimentar e frequência de consumo de suplementos alimentares: um estudo com atletas de Badminton" (CUNHA; COSTA FILHA; CARVALHO, 2016), publicado na Revista Brasileira de Nutrição Esportiva; Artigo 8 - "Características antropométricas e de desempenho motor de atletas da seleção brasileira de Badminton" (DURIGAN; DOURADO; STANGANELLI, 2013); Artigo 9 - "Desempenho motor de atletas de Badminton adolescentes" (BROWNE et al., 2013); Artigo 10 - "Análise da quantificação das ações motoras e da estrutura temporal no Badminton" (FONTES et al. 2014); Artigo 11 - "Características antropométricas e físicas da seleção brasileira de Badminton júnior" (MASCARA; CHIMINAZZO; OLIVEIRA, 2015); Artigo 12 - "Desempenho no salto vertical e utilização da energia elástica em jogadores de Badminton" (LIMA et al., 2018), publicados na Revista Brasileira de Prescrição e Fisiologia do Exercício; e, Artigo 13 - "Adaptações de estratégias e recursos como auxilio à prática do Badminton às crianças com deficiência intelectual" (OLIVEIRA; FAUSTINO; SEABRA JÚNIOR, 2014), publicado na Revista Eletrônica Gestão \& Saúde.

Com a leitura dos artigos na íntegra, optou-se por analisá-los a partir dos princípios da análise de conteúdo, seguindo o método de Richardson (2017). O autor propõe a análise por meio de temáticas, fundamentadas em categorizações. Isso quer dizer que, foram criadas categorias para determinadas temáticas em comum e por meio delas podem existir classificação de assuntos levando assim o pesquisador a isolar temas de registro e retirar partes que irá auxiliar na interpretação dos resultados do estudo. Assim, emergiram-se as seguintes categorias: a) Mapeamento dos autores da produção selecionada sobre Badminton; b) Procedimentos metodológicos abordados na produção selecionada sobre Badminton; e, c) Principais temáticas das pesquisas selecionadas sobre Badminton. Ressalta-se também que, para melhor organização, adotou-se a frequência (f) e porcentagem (\%) para elucidar os achados que apresentaram similaridades ou disparidade.

\section{RESULTADOS}

\section{Mapeamento dos pesquisadores brasileiros sobre Badminton}

A partir do levantamento dos artigos que tratam sobre a temática do Badminton, nas bases de dados selecionadas, foi possível identificar os autores responsáveis pelas produções e as regiões a que pertencem no cenário brasileiro (Tabela 1).

Tabela 1. Quantificação de Autores de acordo com a produção científica e região.

\begin{tabular}{lccccc}
\hline & \multicolumn{5}{c}{ Regiões } \\
\cline { 2 - 6 } Produções & Sul & Sudeste & $\begin{array}{c}\text { Centro } \\
\text { Oeste }\end{array}$ & Nordeste & Norte \\
\hline Artigo 1 & 1 & 3 & 0 & 0 & 0 \\
Artigo 2 & 1 & 4 & 0 & 0 & 0 \\
Artigo 3 & 0 & 0 & 0 & 6 & 0 \\
Artigo 4 & 0 & 1 & 3 & 0 & 0 \\
Artigo 5 & 0 & 2 & 0 & 0 & 0 \\
Artigo 6 & 0 & 0 & 0 & 3 & 0 \\
Artigo 7 & 0 & 0 & 0 & 3 & 0 \\
Artigo 8 & 3 & 0 & 0 & 0 & 0 \\
Artigo 9 & 0 & 0 & 3 & 4 & 0 \\
Artigo 10 & 0 & 3 & 0 & 0 & 0 \\
Artigo 11 & 0 & 2 & 0 & 0 & 0 \\
Artigo 12 & 0 & 0 & 0 & 7 & 0 \\
Artigo 13 & 0 & 3 & 0 & 0 & 0 \\
TOTAL & $\mathbf{5}$ & $\mathbf{1 8}$ & $\mathbf{6}$ & $\mathbf{2 3}$ & $\mathbf{0}$ \\
\hline
\end{tabular}

Conforme a Tabela 1, em relação a região geográfica, destaca-se que o Nordeste tem a maior concentração de pesquisadores sobre o Badminton $(f=23)$, seguido da região Sudeste $(f=18)$, Centro-Oeste $(f=6)$ e Sul $(f=5)$. Nota-se, a partir dos artigos analisados, a ausência de pesquisadores da região Norte.

Em relação às instituições vinculadas aos pesquisadores, destaca-se a Universidade Estadual de Campinas (UNICAMP) 
como a instituição com maior incidência de pesquisadores sobre o assunto, seguido da Faculdade Santo Agostinho (FASA), Centro Universitário UniMetrocamp (METROCAMP), Universidade Federal do Mato Grosso (UFMT), Universidade Federal da Paraíba (UFPB), Universidade Cruzeiro do Sul (UNICSUL), Universidade Paranaense (UNIPAR), Universidade Estadual de Londrina (UEL), Pontifícia Universidade Católica Campinas (PUC), Universidade Federal do Maranhão (UFMA), Universidade Estadual Paulista (UNESP), Universidade Federal do Piauí (UFPI), Universidade Católica de Brasília (UCB), Instituto Federal do Piauí (IFPI) e Badminton Blumenau Clube (BBC). Um possível indicativo para a constatação do maior número de pesquisadores sobre a temática na UNICAMP, é que a mesma, por meio do seu Departamento de Educação Física, busca realizar torneios da modalidade em suas dependências, incentivando a prática e o interesse em difundir o Badminton e/ou Parabadminton.

Ao analisar os sites oficiais das instituições elencadas, em especial das Universidades, constatou-se a oferta de projetos de extensão para a comunidade ligados aos esportes de raquete e, principalmente, ao Badminton. A criação de projetos extensionistas sobre a temática, impulsiona o aumento da visibilidade ao esporte, assim como o crescimento do número de praticantes, visto que, segundo Lima, Ferreira Neto e Pompeu (2019), a extensão nas instituições de ensino superior tem entre seus propósitos a transformação no seu entorno, aproximando instituição de ensino e comunidade. Ainda nessa perspectiva, destaca-se que a UFMT foi a primeira Universidade do Brasil a receber certificação mundial de Badminton, pela World Badminton Federation (BWF), demonstrando a dedicação da referida instituição para com a modalidade.

Com relação a formação inicial dos pesquisadores sobre o Badminton, ao analisar os Currículos Lattes, percebe-se que a maioria é formada em Educação Física, tendo também alguns formados em Fisioterapia, Medicina ou Nutrição. Foi notório que, ao menos um dos autores em cada artigo selecionado mantém elo de ligação com a modalidade de Badminton, seja como técnico desportivo, membro da Confederação Brasileira de Badminton, Fisioterapeuta da Seleção Brasileira ou coordenador de projetos de extensão voltados para a modalidade convencional ou adaptada.

Deste modo, pode-se indicar que o interesse pelo contexto do Badminton se apoia em experiências prévias, formais ou informais, que fomentam os profissionais ligados à área a criar iniciativas de pesquisas, trazendo a luz novos conhecimentos sobre temáticas pouco exploradas e os benefícios que a criação de parcerias entre universidades, pesquisadores e profissionais da área podem conferir a expansão das pesquisas e avanços científicos para a área.

Dentre os autores dos artigos analisados, destaca-se João Guilherme Cren Chiminazzo, o qual está presente em três, dos 13 artigos selecionados. Ressalta-se que o pesquisador realiza estudos relacionados aos esportes de raquete, mais precisamente o Badminton e o Tênis de Campo. O fato de estar lotado como docente em uma Universidade no estado de São Paulo pode ser considerado um dos motivos do protagonismo da região Sudeste, juntamente com o Nordeste, em pesquisas relacionadas com o Badminton.

Ainda sobre o currículo dos pesquisadores, destaca-se que há autores que buscam direcionar suas pesquisas ao esporte adaptado, principalmente as diversas modalidades paralímpicas, incluindo o Parabadminton, resultando em pesquisas que buscam analisar a questão do Badminton de maneira adaptada.

Em sua maioria, os autores estão ligados a projetos de extensão em que a modalidade esportiva Badminton se faz presente, e tais projetos se configuram como campo de pesquisa, direcionando os olhares para a modalidade, e buscando avanços, sejam eles relacionados as metodologias de ensino, as consequências do esporte para a área cognitiva, avaliações posturais, busca de melhores rendimentos em treinamentos, suplementação alimentar e o desempenho motor.

Dentre as produções científicas selecionadas nessa pesquisa, duas pesquisas tiveram como foco o ensino da modalidade na escola, Artigo 4 e Artigo 13. Nesse sentido, pode-se verificar que os pesquisadores dessas produções possuem afinidade com o ambiente escolar e que voltam suas pesquisas a esta área, seja na perspectiva da educação de maneira geral ou com foco para a inclusão escolar. Além disso, no Artigo 4, destaca-se que o trabalho possui a participação de um dos membros da Confederação Brasileira de Badminton, o qual direciona suas produções de conhecimento e pesquisas de pós-graduação para a temática do Badminton.

É importante ressaltar que dentre os autores dos artigos analisados, encontram-se pesquisadores que dedicam seus estudos integralmente ao esporte Badminton, um deles é Presidente da Associação Viva o Badminton e recebe fundos de pesquisa da Badminton World Federation, já foi técnico de várias equipes de Badminton e hoje coordena projetos de pesquisa e extensão com o treinamento da modalidade.

\section{Procedimentos metodológicos adotados nas pesquisas sobre Badminton}

A partir do fichamento dos 13 artigos, observa-se o predomínio de um método de pesquisa, e à adoção de diferentes tipos e técnicas de coleta e análise. Alguns deles se assemelham em relação aos seus instrumentos de coleta de dados e/ou análise das informações, bem como outros estudos partem de vertentes teóricas e metodológicas diferentes (Tabela 2).

Tabela 2. Procedimentos metodológicos adotados nas pesquisas selecionadas.

\begin{tabular}{llll}
\hline $\begin{array}{c}\text { Tipo de } \\
\text { pesquisa }\end{array}$ & $\mathbf{f ( \% )}$ & \multicolumn{1}{c}{ Instrumento de Coleta } & $\mathbf{f ( \% )}$ \\
\hline & & $\begin{array}{l}\text { Testes Físicos de Desempenho Neuro- } \\
\text { musculares } \\
\text { Planilha de Scout }\end{array}$ & $5(45,4 \%)$ \\
Quantitativa & $11(84,6 \%)$ & $\begin{array}{l}\text { Filmagem de Programa de Intervenção/ } \\
\text { Aulas }\end{array}$ & $1(27,3 \%)$ \\
& & $\begin{array}{l}\text { Imagem por Câmera Fotográfica } \\
\text { Entrevista Estruturada }\end{array}$ & $1(9,1 \%)$ \\
\hline \multirow{2}{*}{ Qualitativa } & $2(15,4 \%)$ & Filmagem dos Jogos & $1(9,1 \%)$ \\
\hline
\end{tabular}

Fonte: os autores

A partir dos dados apresentados na Tabela 2, nota-se a sobreposição das pesquisas quantitativas relacionadas ao Bad- 
minton, totalizando 11 pesquisas com um percentual de $84,6 \%$. Tais pesquisas quantitativas, apresentam características de mensuração dos seus achados por meio de testes e protocolos, as quais, muitas vezes, se dedicam a levantar frequências em que determinado fenômeno e sua intensidade ocorrem (SILVA; SIMON, 2005). Referindo-se as pesquisas na temática do Badminton, suas diferenciações se apresentam na diversidade dos instrumentos de coleta utilizados, determinando assim o direcionamento de seus achados. Algumas pesquisas aqui encontradas, se correlacionam e outras se distinguem quanto ao instrumento de coleta.

Como exemplificação das correlações, grande parte das pesquisas quantitativas adotaram seu instrumento de coleta, com testes de desempenho físico e neuromuscular. Sendo encontrados nos Artigos 5, 8, 9, 11 e 12 (LOUREIRO JUNIOR; FREITAS, 2012; DURINGAN; DOURADO; STANGANELLI, 2013; BROWNE et al., 2013; MASCARA; CHIMINAZZO; OLIVEIRA, 2015; LIMA et al., 2018). Os estudos em sua metodologia, tinham sua objetividade em identificar valores antropométricos e a potência de diversos membros corporais dos atletas. Além de realizarem o tratamento dos dados, obtidos por meio de equações, cálculos e softwares estatísticos.

Obtemos ainda três pesquisas quantitativas utilizando o instrumento de coleta por meio da planilha de Scout, caracterizando o percentual de $27,3 \%$ dos achados. Na planilha de Scout os pesquisadores coletaram vários registros de informações com base na análise do jogo. Esse processo é evidenciado nos artigos 1, 2 e 10 (CHIMINAZZO et al., 2017; STRAPASSON et al., 2017; FONTES et al., 2014). Nestas pesquisas os acréscimos com analises estatísticas contribuem para a fidedignidade de seus resultados.

Já no Artigo 4, este é retratado por diferentes métodos de ensino do Badminton no contexto escolar. Aburachid et al. (2019) propõem um estudo pré-experimental de tentativa única, que foi aplicado a três grupos, compostos por 16 escolares e um professor para cada grupo, objetivando avaliar a frequência de ocorrências nas aulas e não o desempenho dos avaliados. Quanto a aplicação, cada grupo recebeu de forma não intencional um programa de intervenção com aulas previamente elaboradas, e os professores foram treinados previamente para aplicação das aulas. Com relação ao tempo de intervenção, somaram-se 17 sessões, com 50 minutos cada, totalizando aproximadamente 850 minutos de aplicação. Utilizou-se a teoria do desenvolvimento ecológico como forma de categorizar o treino, uma vez que essa possibilita a mensuração da frequência e duração dos objetivos da análise (segmento da aula, quantidade de participantes e delimitação espacial da atividade, distribuição e condição da tarefa, organização da prática e organização da técnica) (ABURACHID et al., 2019). No que se refere a análise estatística dos dados, os autores optaram por utilizar a frequência absoluta e tempo para apresentar os dados das atividades, assim como verificar o tempo em minutos quanto a estimulação de cada indicador. Os pesquisadores, assim como em outros estudos citados, fizeram uso do Excel para organizar as planilhas de cálculo e usaram o SPSS 20.0 para análise estatística, considerando valor de $p \leq 0,05$.

Por sua vez, o Artigo 3 (DANTAS et al., 2014) fez uso de imagens com câmera fotográfica. Aplicando um plano de intervenção junto a 23 atletas da seleção piauiense de Badminton, realizando sessões com método de Stretching Global Ativo, contendo três ações posturais. Para análise postural, fizeram uso da biofotogrametria nas visões anterior e posterior, por meio do software Corel Draw X-6. E para análise estatística foi usado o teste " $\mathrm{t}$ " student, considerando como nível de significância $p<0,05$.

No Artigo 7 (CUNHA; COSTA FILHA; CARVALHO, 2016), os pesquisadores utilizaram como instrumento de coleta, à aplicação de um roteiro de entrevista estruturada em 11 atletas com faixa etárias entre 12 a 19 anos da seleção piauiense de Teresina-Piauí. A objetividade do roteiro de entrevista, se deu por meio da avaliação do consumo de frequência alimentar e o uso de suplementos nutricionais durante a realização dos treinos. Os dados obtidos a partir deste roteiro, foram digitalizados e interpretados através do software Statistical Package for the Social Science (SPSS) versão 9.0 e o software Excel.

Com relação aos estudos sobre o Badminton de cunho qualitativo, estes representam apenas $15,4 \%$ (2 f) do total de estudos elencados para a presente pesquisa. Deste modo, enquadraram-se nesta caracterização, os Artigos 6 e 13 (GOMES-DA-SILVA; SOUSA-CRUZ; ARRUDA, 2019; OLIVEIRA, FAUSTINO; SEABRA-JÚNIOR, 2014). Estes também se correlacionam por utilizarem a metodologia observacional, porém se distinguem em seus instrumentos de coletas utilizados. No caso do Artigo 6, os pesquisadores optaram por meio de filmagens para observar a final das Olímpiadas Escolares- Etapa 12-14 anos, entre as atletas do estado de Piauí e São Paulo, afim de enfatizarem as ações motrizes ocorridas no jogo, ao qual descreveram o ambiente de funcionamento, identificando conhecer a lógica interna do badminton. Para isto necessitou de programas com ferramentas de recortes das imagens/cenas e o selecionamento das mesmas.

No outro estudo, Artigo 13, (OLIVEIRA; FAUSTINO; SEABRA JÚNIOR, 2014), ressalta-se o método interventivo, realizado na Educação Infantil da APAE de Presidente Prudente (SP). Os pesquisadores aplicaram 17 aulas com duração de 50 minutos, com a finalidade de trabalhar o histórico e as habilidades motoras do badminton. Para isto, foram divididas tais habilidades em estabilizantes, locomotoras e manipulativas. Ressalta-se que o estudo não retrata como os registros da pesquisa se constituíram, ficando subentendido que a análise da pesquisa aconteceu por meio dos planos de aula e observação dos professores/pesquisadores.

\section{Enfoque temático abordado na produção científica sobre Badminton}

Por meio da análise dos artigos selecionados acerca do Badminton, emergiram-se temáticas principais e secundárias que configuram o enfoque dado às produções científicas apresentadas no cenário brasileiro (Figura 2). Nota-se que as pesquisas versam suas análises da modalidade como esporte de rendimento ou como esporte educacional.

No grupo de artigos direcionados para a temática do esporte de rendimento, foi possível agrupar os artigos em três temáticas secundárias: desempenho técnico-tático (GOMESDA-SILVA; SOUZA-CRUZ; ARRUDA, 2019; CHIMINAZZO et al., 2017; STRAPASSON et al., 2017); nutrição esportiva (CUNHA; COSTA FILHA; CARVALHO, 2016); e desempenho motor (LIMA et al., 2018; MASCARA; CHIMINAZZO; OLIVEIRA, 2015; DANTAS et 
al., 2014; FONTES et al. 2014; BROWNE et al., 2013; DURIGAN; DOURADO; STANGANELLI, 2013; LOUREIRO JÚNIOR; FREITAS, 2012).

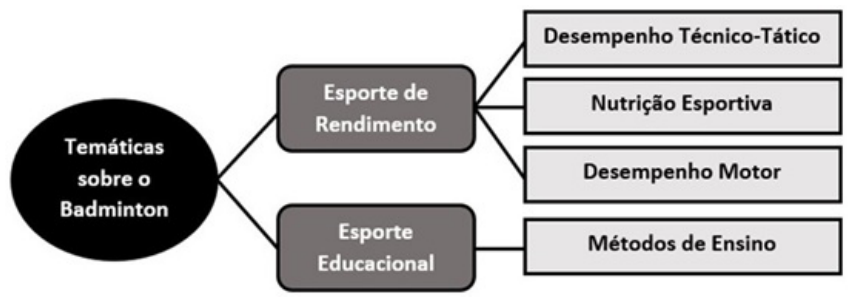

Figura 2. Temáticas encontradas nas pesquisas sobre o Badminton. Fonte: os autores.

Sobre a temática secundária do desempenho técnico-tático, Costa e Nascimento (2004) afirmam que a técnica é uma forma de alcançar o melhor resultado de forma rápida e efetiva, a qual é composta por etapas verbais e procedimentais, isto é, a técnica é um aspecto de preparação pelo qual se obtém o rendimento. Já a tática é um processo de tomada de decisão, no qual, o atleta, dentro do jogo faz a ligação entre o conhecimento que possui sobre a modalidade, adversário, quadra, entre outros, com o desenvolvimento e dinâmica da partida (COSTA; NASCIMENTO, 2004).

Com isso, nota-se que no Artigo 2, Chiminazzo et al. (2017) fazem uma análise de jogos de campeonatos nacionais e estaduais de Badminton, no qual eles puderam avaliar os jogadores, com o intuito de verificar a constância de pontos vencidos e os erros não forçados em jogos com vitória e derrota. E como resultado autores concluem que os atletas que erram menos têm maior chance de vitória.

No Artigo 6, Gomes-da-Silva, Sousa-Cruz e Arruda (2019) seguem essa mesma linha de análise, ao avaliarem as finais de um campeonato escolar de Badminton, porém fazendo uso de uma metodologia diferente dos autores do Artigo 2. Baseados na praxiologia motriz, os autores se debruçam a compreender o sistema de ação dos jogadores (a partir dos Universais), dando ênfase na relação práxica de contracomunicação. Quanto aos resultados encontrados pelos autores, destaca-se que o Badminton, por ser um jogo de muita agilidade, mostrou-se dependente da leitura, análise e previsão dos movimentos adversários, bem como uma tomada de decisão rápida para evitar o erro e induzir o erro ao adversário. Para os pesquisadores, o movimento de contracomunicação que ocorre durante o jogo possibilita essa leitura, análise e previsão ao atleta. Além disso, os autores afirmam que toda dinâmica do jogo foi iniciada na posição de expectativa, possibilitando uma maior concentração e uma melhor leitura do adversário.

Ainda pertencendo a temática esporte de rendimento - desempenho técnico e tático, tem-se o Artigo 1, elaborado por Strapasson et al. (2017), que aborda a prática de esportes paralímpicos, que no caso é o Parabadminton. O Parabadminton foi criado com o objetivo de incluir pessoas com deficiência na prática do esporte, assim apresentando as mesmas regras do esporte convencional, sofrendo apenas adaptações quanto a deficiência de cada atleta, ao tamanho da quadra e equipamentos adicionais (muletas e/ou cadeira de rodas) (STRAPASSON;
DUARTE; PEREIRA, 2015). Considerando então esses fatores, Strapasson et al. (2017) buscaram apresentar uma análise dos fundamentos que apareceram nas finais do campeonato mundial de Parabadminton, e para essa análise eles usam do scout técnico. Como resultado das análises, destaca-se que o atleta que teve menos erros foi quem ganhou, que o local mais efetivo para a marcação de pontos nas partidas foi a parte frontal da quadra e os principais erros cometidos pelos atletas foram o lançamento das petecas para fora da quadra adversária.

Sobre a temática secundária da nutrição esportiva, tem-se o Artigo 7, desenvolvido por Cunha, Costa Filha e Carvalho (2016). Para os autores, quando se aborda a vida do atleta de alto rendimento, a alimentação adequada é importante para manutenção da saúde, controle de composição corporal e do peso, resultando em bom rendimento nos treinos e nos campeonatos. Sendo assim, nesse estudo, os autores buscaram avaliar os hábitos alimentares de atletas adolescentes de Badminton, o qual apresentou um resultado um tanto quanto preocupante, pois foi possível constatar que a alimentação não atendia as necessidades nutricionais dos jovens atletas, inviabilizando assim a aquisição de um melhor desempenho na prática, bem como consequências para o desenvolvimento nessa fase da vida. E é por isso que muitos acabam optando por uma suplementação alimentar para suprir esse déficit e melhorar o desempenho esportivo.

Quanto a temática secundária do desempenho motor, agruparam-se os Artigos 3, 5, 8, 9, 10, 11 e 12 os quais focam seus olhares para a dinâmica do movimento ao jogar o Badminton. No trabalho publicado por Loureiro Júnior e Freitas (2012) (Artigo 5), os pesquisadores buscaram comparar o comportamento neuromotor em uma tarefa isolada entre atletas experientes e atletas menos experientes praticantes da modalidade. Como resultado do estudo, foi possível identificar que os jogadores com mais experiência tendem a ter uma reação a um estímulo visual mais rápida que os menos experientes, porém, ambos atletas têm movimentos em tempos similares.

Em paralelo ao Artigo 5, o Artigo 10, proposto por Fontes et al. (2014), segue uma linha semelhante de análise, porém, sem a comparação entre os atletas. No caso deste artigo, os autores fazem uma análise dos jogos de atletas adultos da categoria máscula e feminina. Com objetivo de analisar quais ações motores estão mais presentes nos jogos da competição nacional. Nos resultados encontrados pelos autores, destaca-se que seguem no mesmo caminho que a literatura, ou seja, não apresentam uma diferença significativa entre os gêneros, mas deve-se respeitar as individualidades biológicas de cada atleta ao designar um treinamento esportivo.

Nessa mesma temática, encontram-se estudos que se assemelham devido as metodologias utilizadas, como os Artigos 8, 9 e 11, propostos por Durigan, Dourado e Stanganelli (2013), Browne et al. (2013), e Lima et al. (2018), respectivamente. Os três estudos retratam a avaliação antropométrica de atletas e uma sequência de testes de desempenho motor, como testes de velocidade, força de membros superiores e inferiores, agilidade e resistência. Sendo que o que diverge um pouco mais dentre os três artigos é o Artigo 11, elaborado por Lima et al. (2018), já que os autores se restringiram em avaliar apenas a potência de saltos dos atletas e elasticidade dos mesmos, para além das medidas antropométricas. 
Já na produção de Dantas et al. (2014), os autores fazem uso de um método que trabalha com a reeducação postural, conhecido por Stretching Global Ativo (SGA) proposto por Philippe Souchard em 1995, que consiste em uma série de alongamentos que visam melhorar o desempenho muscular e evitar lesões, possibilitando um fortalecimento muscular, melhorando a postura, entre outros benefícios. Assim, ao utilizar esse método em jogadores de Badminton os pesquisadores concluíram que o SGA promoveu uma melhora na performance dos atletas, prevenindo possíveis lesões como a hiperlordose lombar, que é a mais comum em praticantes dessa modalidade.

Sabe-se que o esporte de alto nível exige muito dos atletas, e, muitas vezes, tais exigências podem extrapolar a prática saudável, levando ao desconforto corporal e, em caso de persistência, a limitação da mesma. Dentre as exigências desprendidas no esporte, a postura é uma delas, a qual se não cuidada pode ser prejudicial, pois o corpo quando exposto a uma prática associada a muitas repetições e exigência de força em direções diferentes, acaba se expondo a altas cargas mecânicas, o que pode sobrecarregar a coluna (DETANICO et al., 2008; BARTLETT, 1999).

No caso da prática do Badminton de alto nível, nota-se que este esporte acaba por exigir muito de seus praticantes, visto que é um dos jogos de raquete mais rápidos do mundo, o que exige um desgaste físico, já que as partidas podem ter mais de uma hora de duração, quanto mental, pois os atletas devem pensar rápido nos movimentos que devem fazer e ter uma boa acurácia temporal e espacial para posicionar a raquete a fim de conduzir a peteca para o local planejado (DANTAS et al., 2014; LOUREIRO JÚNIOR; FREITAS, 2012).

Por fim, no grupo temático nomeado de esporte educacional, tem-se como temática secundária os métodos de ensino (ABURACHID, et al., 2019; OLIVEIRA; FAUSTINO; SEABRA JÚNIOR, 2014). Essa categoria se coloca como interessante, uma vez que, pode retratar a tematização dos esportes na Educação Física escolar, traçando uma relação delicada, ao desconstruir a imagem que o esporte tem na sociedade, transcendendo o valor a ele atribuído de alto rendimento, apenas. Tal olhar reforça a ideia de ressignificação da aprendizagem dos esportes na escola, ao atribuir novas facetas para as modalidades trabalhadas, por meio de diferentes métodos de ensino, aplicados nas aulas desse componente curricular (TANI et al., 2013).

E é isso que o Artigo 4 se propõe a apresentar. Aburachid et al. (2019) trazem como objetivo demonstrar que o Badminton pode ser ensinado por meio de métodos diferentes nas aulas de Educação Física. No trabalho os autores utilizaram três métodos de ensino ao longo de suas aulas: o método analítico, o método global e o integrativo. As aulas foram elaboradas e aplicadas por três professores da área, as quais foram analisadas a partir da forma como a aula acontecia e da postura do professor ao longo da intervenção. No estudo, evidenciou-se que cada método trouxe resultados positivos e negativos, pois o método analítico serviu como forma de ensino dos fundamentos individuais com práticas em blocos e partes fracionadas sem oposição; o método global consistiu em uma maior parte da aula voltada para fundamentos técnicos-táticos aplicados no jogo; e, o método integrativo, consistiu em conteúdos técnico-táticos, diversificando a prática quanto a quantidade de jogadores e quanto a aplicação da técnica por meio de jogos diversificados.
Seguindo esse viés, o Artigo 13 (OLIVEIRA; FAUSTINO; SEABRA JÚNIOR, 2014), apresenta estratégias de ensino e recursos pedagógicos para aplicação do Badminton de forma adaptada a alunos com deficiência intelectual. Por meio de uma pesquisa interventiva, o trabalho foi desenvolvido a partir da aplicação de aulas para alunos da Associação de Pais e Amigos dos Excepcionais (APAE) da cidade de Presidente Prudente/SP. Para organizar as ações desenvolvidas nas aulas, a partir da dimensão motora, os pesquisadores optaram por dividir as habilidades do Badminton em estabilizantes, locomotoras e manipulativas. Por fim, os autores concluem que foi de suma importância trabalhar essa modalidade tendo em vista que possibilita uma aprimoração do processo de ensino-aprendizagem. Além disso, conforme Oliveira, Faustino e Seabra Júnior (2014), o esporte adaptado possibilitou a inclusão social, ganhos físicos, psíquicos e emocionais, o que pode desencadear na pessoa com deficiência uma maior autoestima, confiando mais em si mesmo, encarando assim da melhor forma possível a sociedade e as dificuldades que se fazem presentes no dia-a-dia.

\section{CONCLUSÃO}

A análise da produção científica sobre o Badminton permite verificar que, nas bases analisadas, são poucas as produções científicas sobre a temática no contexto brasileiro, uma vez que a modalidade ainda é pouco difundida no país. Destaca-se que a Revista Brasileira de Prescrição e Fisiologia do Exercício foi o periódico que mais apresentou artigos sobre a temática.

Frente a esse panorama, ao fazer o mapeamento dos autores sobre Badminton, constata-se que todas as produções científicas selecionadas, contam com pesquisadores que possuem vínculo, identificação ou relação direta com a modalidade, seja ela convencional ou paralímpica, principalmente, em seu formato de rendimento. As produções são desenvolvidas em sua maioria nas regiões Nordeste e Sudeste, sendo a UNICAMP a instituição que possui mais pesquisadores ligados a temática, com projetos de extensão da modalidade na vertente convencional e adaptada. Ressalta-se que a região Norte não possui pesquisadores que publicam sobre o Badminton nas bases investigadas.

Sobre os procedimentos metodológicos abordados, constatou-se que a maioria dos estudos adota o método quantitativo, utilizando-se de testes físicos, por meio de protocolos de avaliação, para coleta dos dados. Nota-se que a maioria das pesquisas utilizam dos atletas de Badminton como lócus de investigação, mas não se detém a compreender a modalidade como fenômeno social e cultural.

Em relação as temáticas, emergiram duas categorias principais, o esporte de rendimento e o esporte educacional. No esporte de rendimento a maioria das pesquisas voltaram seus olhares para o desempenho motor desprendido pelos atletas, relacionando a necessidade da técnica e do movimento durante os jogos, desvelando que o interesse investigativo sobre o Badminton ainda se constitui para o movimento técnico. Tal interesse, pode justificar o porquê de poucos estudos sobre o Badminton a partir do contexto do esporte educacional. Sobre a categoria do esporte educacional, ressalta-se que as duas pesquisas retratam o ensino da modalidade, seja por meio de métodos diversificados ou como meio de inclusão social para pessoas com deficiência.

Destaca-se que, a partir do mapeamento e análise das pes- 
quisas sobre a temática, há uma carência de pesquisas que abordem a prática do Badminton na área da saúde e do lazer (esporte participação). Isso se confirma, uma vez que não foi encontrado nenhuma produção que tratasse sobre o Badminton dentro desse contexto.

\section{REFERÊNCIAS}

ABURACHID, L. M. C.; SILVA, S. R.; ARAÚJO, N. D.; GRECO, P. J. Badminton: possibilidades de ensino aplicadas ao contexto da educação física escolar Journal of Physical Education, Maringá, v. 30, n. 1, p. 1-12, 2019.

ANVERSA, A. L. B.; FLORES, P. P. Esportes com rede divisória. In: FLORES, P. P. (Org.). Esportes Complementares. Maringá: UniCesumar, 2019. p. 92-139.

BARTLETT, R. Injuries in sport: how the body behaves under load.: how the body behaves under load. In: BARTLETT, R. (Org.). Sports Biomechanics: preventing injury and improving performance. preventing injury and improving performance. London: Taylor \& Francis E-library, 1999. p. 36-65.

BENTO, J. Em nome do desporto. In: GAYA, A. C. A. (Org.). Educação Física: ordem, caos e utopia. Casa da Educação Física: Belo Horizonte, 2014. p. 13578.

BROWNE, R. A. V.; SALES, M. M.; LIMA, S. F. C.; SANTOS, L. C. S.; ROCHA FILHO, J. B.; MACÊDO, T. A.; OLHER, R. R. V. Desempenho motor de atletas de Badminton adolescentes. Revista Brasileira de Nutrição Esportiva, São Paulo, v. 7, n. 38, p. 115-22, 2013.

CBBD. Confederação Brasileira de Badminton. Jogos Olímpicos. Disponíve em: <http://www.badminton.org.br/>. Acessado em: 16 de junho de 2020

CHIMINAZZO, J. G. C.; FERREIRA, R.; CASTANHO, G. K. F.; BARREIRA, J.; FERNANDES, P. T. Errar menos para ganhar mais: uma análise no badminton. Revista Brasileira de Ciência e Movimento, Brasília, v. 25, n. 2, p. 115-21, 2017.

COSTA, L. C. A.; NASCIMENTO, J. V. O ensino da técnica e da tática: novas abordagens metodológicas. Revista da Educação Física, Maringá, v. 15, n 2, p. 49-56, 2004

CROSSETTI, M. G. O. Revisão integrativa de pesquisa na enfermagem: o rigor científico que lhe é exigido. Revista Gaúcha de Enfermagem, Porto Alegre, v. 33, n. 2, p. $8-9,2012$

CUNHA, L. M. S.; COSTA FILHA, L. C. G. I.; CARVALHO, L. M. F. Hábito alimentar e frequência de consumo de suplementos alimentares: um estudo com atletas de badminton. Revista Brasileira de Nutrição Esportiva, São Paulo, v. 10, n. 60 , p. $673-8,2016$

DANTAS, S. V.; NEVES, I. S. F.; MOTA, D. M.; MARQUES, C. O.; SOUSA, F. A. N.; LEAL, S. S. Avaliação das alterações posturais de atletas de badminton após stretching global ativo. Conscientiae Saúde, São Paulo, v. 2, n. 13, p. 211-7, 2014

DETANICO, D.; REIS, D. C.; CHAGAS, L.; SANTOS S. G. Alterações posturais, desconforto corporal (dor) e lesões em atletas das seleções brasileiras de hóquei sobre a grama. Revista da Educação Física, Maringá, v. 19, n. 3, p. 423-30, 2008.

DURIGAN, J. Z.; DOURADO, A. C.; STANGANELLI, L. C. R. Características antropométricas e de desempenho motor de atletas da seleção brasileira de badminton. Revista Brasileira de Prescrição e Fisiologia do Exercício, São Paulo, v. 7, n. 38, p. 161-6, 2013.

FONTES, T. T.; DOBRÁNSZKY, I. A.; CHIMINAZZO, J. G. C.; MORAIS, A. M. Análise da quantificação das ações motoras e da estrutura temporal no badminton. Revista Brasileira de Prescrição e Fisiologia do Exercício, São Paulo, v. 8, n. 50, p. 782-6, 2014.

GIL, A. C. Como elaborar projetos de pesquisa. 4. ed. São Paulo: Atlas, 2002.

GINCIENE, G.; ABURACHID, L. M. C. Badminton. In: GONZÁLEZ, F. J.; DARIDO S. C.; OLIVEIRA, A. A. B. Esportes de marca e com rede divisória ou muro/ parede de rebote: badminton, peteca, tênis de campo, tênis de mesa, voleibol, atletismo. 2. ed. Maringá: Eduem, 2017. p. 65-109.

GOMES-DA-SILVA, P. N.; SOUSA-CRUZ, R. W.; ARRUDA, E. P. S. Entre lance e a chance: lógica interna numa final de badminton. Motrivivência, Florianópolis, v. 31, n. 58, p. 1-19, 2019.

GONZÁLEZ, F. J.; DARIDO, S. C.; OLIVEIRA, A. A. B. Esportes de marca e com rede divisória ou muro/parede de rebote: badminton, peteca, tênis de campo, tênis de mesa, voleibol, atletismo. 2. ed. Maringá: Eduem, 2017.

LIMA, K. C. G.; RIBEIRO, S. L. G.; CABRAL, C. O. M.; ALVARES, P. D.; RODRIGUES, N. T.; CABIDO, C. E. T.; SANTOS, M. A. P. Desempenho no salto vertical e utilização da energia elástica em jogadores de Badminton. Revista
Brasileira de Prescrição e Fisiologia do Exercício, São Paulo, v. 12, n. 80, p. 1193-9, 2018.

LIMA, M. X. A.; FERREIRA NETO, M. N.; POMPEU, R. M. Projeto de extensão no ensino superior como prática de responsabilidade social. Revista Gestão e Avaliação Educacional, Santa Maria, v. 8, n. 17, p. 1-12, 2019.

LOUREIRO JÚNIOR, L. F. B.; FREITAS, P. B. Influência do nível de desempenho de jogadores de badminton em aspectos neuromotores durante uma tarefa de apontar um alvo. Revista Brasileira de Medicina do Esporte, São Paulo, v. 18, n. 3, p. 203-7, 2012

MASCARA, D. I.; CHIMINAZZO, J. G. C.; OLIVEIRA, J. F. Características antropométricas e físicas da seleção brasileira de Badminton júnior. Revista Brasileira de Prescrição e Fisiologia do Exercício, São Paulo, v. 9, n. 53, p. 303-8, 2015.

OLIVEIRA, A. R. P.; FAUSTINO, P. F.; SEABRA JÚNIOR, M. O. Adaptações de estratégias e recursos como auxilio à prática do Badminton às crianças com deficiência intelectual. Revista Eletrônica Gestão \& Saúde, Brasília, v. 5, p. 2234-46, 2014.

RICHARDSON, R. J. Pesquisa social: métodos e técnicas. 4 ed. São Paulo: Atlas, 2017.

SESI. Serviço Social da Indústria. Tênis, Tênis de Mesa e Badminton. São Paulo: Sesi Editora, 2012.

SILVA, D.; SIMON, F. O. Abordagem quantitativa de análise de dados de pesquisa: construção e validação de escala de atitude. Cadernos CERU, São Paulo, v. 2, n. 16, p. 11-27, 2005.

SOUZA, D. L.; SILVA, M. M.; MOREIRA, T. S. O perfil da produção científica online em português relacionada às modalidades olímpicas e paralímpicas. Movimento, Porto Alegre, v. 22, n. 4, p. 1105-20, 2016.

STRAPASSON, A. M.; BAESSA, D. J.; BORIN, J. P.; DUARTE, E. Para-badminton: quantificação dos fundamentos do jogo através do scout. Revista Brasileira de Ciência e Movimento, Brasília, v. 25, n. 2, p. 107-15, 2017.

STRAPASSON, A. M. DUARTE, E. PEREIRA, L. S. O parabadminton no Brasil: um esporte adaptado em ascensão. Revista da Associação Brasileira de Atividade Motora Adaptada, Marília, v. 16, n. 1, p. 19-22, 2015.

TANI, G.; BASSO, L.; SILVEIRA, S. R.; CORREIA, W. R.; CORRÊA, U. C. O ensino de habilidades motoras esportivas na escola e o esporte de alto rendimento: discurso, realidade e possibilidades. Revista Brasileira de Educação Física e Esporte, São Paulo, v. 27, n. 3, p. 507-18, 2013.

\section{AGRADECIMENTOS}

Os autores agradecem as instituições (UNESPAR e UEM) pelo tempo disponibilizado para a produção de conhecimento.

\section{CONFLITO DE INTERESSE}

Os autores do estudo declaram não haver conflito de interesses.

\section{FINANCIAMENTO}

Este estudo não contou com apoio financeiro.

\section{ORCID E E-MAIL DOS AUTORES}

Patric Paludett Flores (Autor Correspondente) ORCID: 0000-0003-4865-7661. E-mail: patricpflores@gmail.com

Ana Luíza Barbosa Anversa ORCID: 0000-0003-4363-3433. E-mail: ana.beah@gmail.com Leonardo Cordeiro de Queiroz ORCID: 0000-0003-1608-3499. E-mail: leonnardoq@gmail.com

Fernando Lazaretti Onorato Silva ORCID: 0000-0002-9247-9869. E-mail: feelazaretti@gmail.com Ariel Arthur Cardoso dos Santos Coutinho ORCID: 0000-0002-1161-6738. E-mail: ra101497@uem.br

Vânia de Fátima Matias de Souza ORCID: 0000-0003-4631-1245.

E-mail:vfmatias@gmail.com 